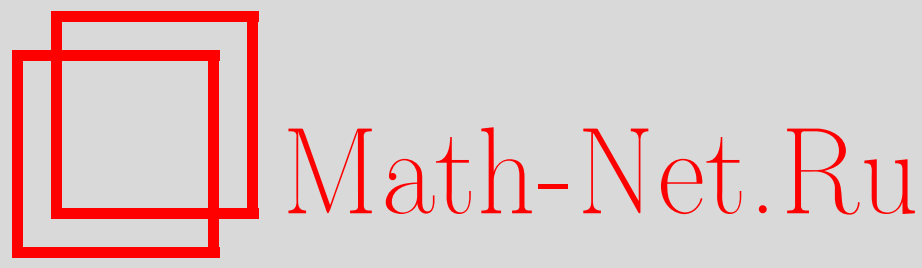

В. С. Мележик, Адиабатическое представление в кулоновской задаче трех тел в пределе объединенного атома: ядерные ширины уровней энергии мезомолекулы $t t \mu$, TMФ, 2016, том 186, номер 1, 76-86

DOI: https://doi.org/10.4213/tmf8978

Использование Общероссийского математического портала Math-Net.Ru подразумевает, что вы прочитали и согласны с пользовательским соглашением http://www . mathnet.ru/rus/agreement

Параметры загрузки:

IP: 54.209 .52 .79

26 апреля 2023 г., 06:15:26

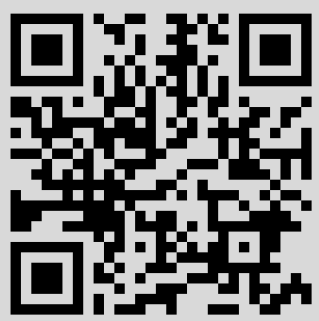




\title{
АДИАБАТИЧЕСКОЕ ПРЕДСТАВЛЕНИЕ В КУЛОНОВСКОЙ ЗАДАЧЕ ТРЕХ ТЕЛ В ПРЕДЕЛЕ ОБЪЕДИНЕННОГО АТОМА: ЯДЕРНЫЕ ШИРИНЫ УРОВНЕЙ ЭНЕРГИИ МЕЗОМОЛЕКУЛЫ $t t \mu$
}

\begin{abstract}
Исследуется асимптотика волновой функции системы трех кулоновских частиц в пределе объединенного атома в адиабатическом представлении задачи трех тел. Этот результат используется при расчете ядерных ширин уровней энергии мезомолекул. Обсуждаются особенности подхода применительно к возбужденным состояниям мезомолекулы $t t \mu$ с ненулевым орбитальным моментом.
\end{abstract}

Ключевые слова: задача трех тел, задача двух кулоновских центров, адиабатическое представление, мюонный катализ, волновая функция мезомолекулы, ядерные реакции в мезомолекулах, астрофизический фактор.

DOI: $10.4213 /$ tmf8978

Посвящается памяти Станислава Петровича Меркуръева

\section{1. ВВЕДЕНИЕ}

Станислав Петрович Меркурьев живо интересовался работами по мюонному катализу и совместно со своими сотрудниками и учениками получил ряд интересных результатов в этой области [1], [2]. В частности, актуальной проблемой в начале 80-х годов прошлого столетия была задача о вычислении волновых функций мезомолекул - связанных состояний системы трех кулоновских частиц (два ядра изотопов водорода и отрицательно заряженный мюон) - в пределе малых межъядерных расстояний. Эти асимптотики были необходимы для расчета скоростей ядерных реакций из различных состояний мезомолекул (ядерных ширин уровней энергии мезомолекул). К тому времени существовали оценки этих величин лишь для основных состояний мезомолекул, полученные в квазиклассическом приближении в работах Зельдовича и Герштейна [3], [4] и Джексона [5]. Однако для количественного описания процессов мюонного катализа в смеси дейтерия и трития требовалось решить

* Объединенный институт ядерных исследований, Дубна, Московская обл., Россия. E-mail: melezhik@theor.jinr.ru

†Университет "Дубна", Дубна, Московская обл., Россия 
задачу о ядерных ширинах уровней энергии возбужденных состояний мезомолекул, поскольку мюонный катализ идет через возбужденные состояния таких систем с ненулевым орбитальным моментом. В свою очередь, это потребовало исследования асимптотики волновых функций возбужденных состояний мезомолекул в пределе малых межъядерных расстояний. Такая задача была решена группой Пономарёва [6] в рамках адиабатического представления задачи трех тел [7], [8]. На основе этих результатов была развита вычислительная схема для расчета ядерных ширин и сдвигов уровней энергии мезомолекул и рассчитаны скорости ядерных реакций из слабосвязанных состояний мезомолекул $d t \mu$ [9], [10] и $d d \mu$ [11], критически важные для описания резонансных процессов мюонного катализа. В работах [9], [10] было показано, что расчет ядерных ширин (скоростей ядерных реакций) и сдвигов уровней энергии мезомолекул можно свести к факторизационному соотношению, представляющему собой произведение константы ядерной реакции (астрофизического фактора) на квадрат волновой функции мезомолекулы в пределе объединенного атома. Астрофизические факторы извлекались при экстраполяции экспериментальных данных по ядерным реакциям "на лету" (ядерным реакциям в свободном пространстве) в область низких энергий, а волновые функции мезомолекулы рассчитывались в адиабатическом представлении задачи трех тел. Результаты этих расчетов впоследствии были подтверждены экспериментально [12]-[16]. В дальнейшем были также выполнены независимые расчеты асимптотик волновых функций мезомолекул в гиперсферическом [17] и вариационном [18], [19] подходах, которые независимо подтвердили результаты работ [6], [9]-[11]. Важный теоретический результат по ядерным реакциям в мезомолекулах изотопов водорода был получен в совместной работе Меркурьева и Пономарёва с соавторами [2], в которой астрофизический фактор для системы $p d \mu$ был получен при решении модифицированных уравнений Фаддеева в гиперсферическом представлении [1].

Относительно недавно в Лаборатории ядерных проблем ОИЯИ был выполнен эксперимент [15], в котором была измерена скорость ядерной реакции $\lambda_{f}^{\exp }=(15.6 \pm$ $2.0) \times 10^{6} \mathrm{c}^{-1}$ в мезомолекуле $t t \mu$ из возбужденных состояний с орбитальным моментом $J=1$, что прекрасно согласуется с определенным нами ранее теоретическим значением $\lambda_{f}^{\text {theor }}=13 \times 10^{6} \mathrm{c}^{-1}[20]-[22]$ и результатом $\lambda_{f}^{\exp }=(15 \pm 2) \times 10^{6} \mathrm{c}^{-1}$, полученным в независимом эксперименте Международной коллаборацией в Лаборатории PSI (Швейцария) [13]. Следует отметить, что в работах [21], [22] приведен лишь результат расчета, выполненного в диссертации автора [20], без подробного изложения использованной теоретической модели, которая существенно отличается от описанных в работах [9], [10] вычислительных схем для несимметричных мезомолекул $(d t \mu$ и $p d \mu)$. Поскольку этот результат оказался неожиданно востребованным и актуальным для современных исследований [16], мне представляется интересным и уместным в статье, посвященной юбилею С.П. Меркурьева, подробно изложить вычислительную схему расчета скоростей ядерных реакций в мезомолекуле $t t \mu$ из возбужденных состояний с орбитальным моментом $J=1$ в адиабатическом представлении задачи трех тел.

\section{2. РАСЧЕТ ЯДЕРНЫХ ШИРИН УРОВНЕЙ ЭНЕРГИИ ВОЗБУЖДЕННЫХ СОСТОЯНИЙ МЕЗОМОЛЕКУЛЫ $Т Т \mu$}

В работах [9], [10] было показано, что скорость ядерной реакции $\lambda_{f}^{J \vartheta}$ из мезомолекулярного состояния с полным орбитальным моментом $J$ и колебательным квантовым числом $\vartheta$ (ядерная ширина $\Gamma_{f}^{J \vartheta}=\hbar \lambda_{f}^{J \vartheta}$ уровня энергии $\left.E_{J \vartheta}\right)$ может быть 
вычислена с хорошей точностью с помощью факторизационного соотношения

$$
\lambda_{f}^{J \vartheta}=K_{L} \int d^{3} r\left|\Psi^{J \vartheta}(\mathbf{r}, \mathbf{R}=0)\right|^{2}
$$

для мезомолекул с различными ядрами (например, $d t \mu$ или $p d \mu$ ), когда асимптотика волновой функции мезомолекулы в пределе малых межъядерных расстояний имеет s-волновой характер по переменной $R$ относительного движения ядер и стремится при $R \rightarrow 0$ (и конечной величине $r$ радиальной координаты мюона) к постоянной величине $\Psi^{J \vartheta}(\mathbf{r}, \mathbf{R}) \sim R^{L} \rightarrow$ const при $L=0$, где $L$ - орбитальный момент относительного движения ядер в мезомолекуле. Здесь

$$
K_{0}=\lim _{v \rightarrow 0} v \sigma_{0}(v) C_{0}^{-2}(v)
$$

- s-волновая константа ядерной реакции синтеза (астрофизический фактор), определяемая s-волновым низкоэнергетическим сечением ядерной реакции синтеза в свободном пространстве $\sigma_{0}(v)$ и гамовским фактором $C_{0}(v)=2 \pi \eta /\left(e^{2 \pi \eta}-1\right)$, где $v-$ относительная скорость движения ядер, $\eta=\alpha c / v, \alpha=1 / 137.036$ - постоянная тонкой структуры, $c$ - скорость света в вакууме.

Однако вопрос о ядерных ширинах возбужденных состояний мезомолекул с одинаковыми ядрами (например, $d d \mu$ или $t t \mu$ ) и $J \neq 0$ требует специального рассмотрения, поскольку в этом случае $L$ может принимать лишь значения $L=|J|,|J \pm 2|, \ldots$ и $\Psi^{J}(\mathbf{r}, \mathbf{R}) \sim R^{J} \rightarrow 0$ при $R \rightarrow 0(r>R)$ [6], [8]. Получим здесь факторизационное соотношение, аналогичное формуле $(1)$, для возбужденного состояния с $J=1$ мезомолеулы $t t \mu$, в котором она образуется в процессах мюонного катализа [16].

Используя оптическую теорему, запишем в борновском приближении парциальное сечение $\sigma_{p}(E)$ для реакции синтеза ядер трития в свободном пространстве:

$$
\sigma_{p}(E)=\frac{4 \pi}{k} \operatorname{Im} f_{p}(\theta=0)=\frac{24 M \pi}{k} \int_{0}^{\infty}\left[\frac{F_{1}(\eta, k R)}{k R}\right]^{2} V_{n}(R) R^{2} d R .
$$

Здесь $f_{p}(\theta)=3 f_{1} P_{1}(\cos \theta), P_{1}(\cos \theta)$ - полином Лежандра первой степени, $k=$ $\sqrt{2 M E} / \hbar, E-$ энергия относительного движения, $M=M_{t} / 2$ - приведенная масса ядер трития, $\left.F_{1}(\eta, k R)\right|_{R \rightarrow 0} \sim k R-$ р-волновая кулоновская функция, регулярная в нуле, и $V_{n}(R)$ - короткодействующий потенциал, описывающий ядерное $t t$-взаимодействие. Воспользовавшись короткодействующим характером ядерных сил $V_{n}(R)$ по сравнению с характерным периодом $T$ осцилляций волновой функции непрерывного спектра относительного движения ядер трития в низкоэнергетическом пределе $(T=2 \pi / k \rightarrow \infty$ при $k \rightarrow 0)$, находим

$$
\begin{aligned}
\left.\sigma_{p}(E)\right|_{k \rightarrow 0} & =\frac{2 M}{k}\left(12 \pi k^{2} C_{1}^{2}\right) \int_{0}^{\infty} R^{4} V_{n}(R) d R= \\
& =\frac{2 M}{k} \lim _{k R \rightarrow 0} \frac{\left|\frac{d}{d R} \psi_{c}^{p}(\eta, k R)\right|^{2}}{\left|\frac{d}{d R} \psi_{c}^{p}(\eta, k R)\right|^{2}} K_{1}^{4} V_{n}(R) d R= \\
& =v^{-1} \lim _{k R \rightarrow 0},
\end{aligned}
$$


где

$$
K_{1}=2 \hbar \int_{0}^{\infty} R^{4} V_{n}(R) d R
$$

- р-волновая константа ядерной реакции, а

$$
\psi_{c}^{p}(\eta, k R)=\left.\frac{1}{k R}(2 L+1) i^{L} e^{i \delta_{L}^{c}} F_{L}(\eta, k R) P_{L}(\cos \theta)\right|_{k R \rightarrow 0}=3 i e^{i \delta_{L}^{c}} k R C_{1}(\eta) P_{1}(\cos \theta)
$$

- р-волновая кулоновская волновая фунция, описывающая относительное движение ядер в непрерывном спектре с орбитальным моментом $L=1, \delta_{L}^{c}$ - кулоновская фаза рассеяния,

$$
C_{1}(\eta)=\frac{1}{3} \sqrt{\frac{2 \pi \eta\left(1+\eta^{2}\right)}{e^{2 \pi \eta}-1}},
$$

а длинная черта над формулой означает интегрирование по углам $\theta$ и $\phi$ вектора $\mathbf{R}$. Вычислить ab initio константу ядерной реакции $K_{1}$ для шестинуклонной системы $t t$ в настоящее время не представляется возможным. Однако ее можно извлечь из экспериментальных данных по дифференциальным сечениям $d \sigma(\theta, E) / d \Omega$ реакций синтеза ядер трития в низкоэнергетической области [23], [24], используя формулы (4) и $(5)$ :

$$
K_{1}=\frac{1}{9} \lim _{v \rightarrow 0} v \sigma_{1}(v) C_{1}^{-2}(\eta)(v M)^{-2}
$$

где

$$
\sigma_{1}(v)=\frac{2 \pi}{3}\left[\frac{d \sigma}{d \Omega}(v, \theta=0)+\frac{d \sigma}{d \Omega}(v, \theta=\pi)-2 \frac{d \sigma}{d \Omega}\left(v, \theta=\frac{\pi}{2}\right)\right] .
$$

Теперь применим факторизационное соотношение для вычисления скорости ядерной реакции $\lambda_{f}^{J \vartheta}$ из возбужденных состояний $(J \vartheta)$ в мезомолекуле $t t \mu$ с $J=1$. При вычислении $\lambda_{f}^{J \vartheta}$ опять воспользуемся короткодействующим характером ядерного $t t$-взаимодействия по сравнению с дальнодействующими кулоновскими силами, связывающими трехчастичную систему $t t \mu$ :

$$
\begin{aligned}
\lambda_{f}^{J \vartheta} & =\int_{0}^{\infty} \int \overline{\left|\Psi^{J \vartheta}(\mathbf{r}, \mathbf{R})\right|^{2}} V_{n}(R) R^{2} d^{3} r d R= \\
& =\lim _{R \rightarrow 0} \int \overline{\left|\frac{d}{d R} \Psi^{J \vartheta}(\mathbf{r}, \mathbf{R})\right|^{2}} d^{3} r \int_{0}^{\infty} R^{4} V_{n}(R) d R= \\
& =K_{1} \lim _{R \rightarrow 0} \int \overline{\left|\frac{d}{d R} \Psi^{J \vartheta}(\mathbf{r}, \mathbf{R})\right|^{2}} d^{3} r=K_{1} \rho_{J \vartheta} .
\end{aligned}
$$

Для вычисления константы

$$
\rho_{J \vartheta}=\lim _{R \rightarrow 0} \int \overline{\left|\frac{d}{d R} \Psi^{J \vartheta}(\mathbf{r}, \mathbf{R})\right|^{2}} d^{3} r
$$

необходимо знать асимптотику волновой функции $\Psi^{J \vartheta}(\mathbf{r}, \mathbf{R})$ связанного состояния трех кулоновских частиц $t t \mu$ в пределе малых межъядерных состояний. Эта задача 
была исследована в адиабатическом представлении задачи трех тел в работе [6]. Следуя [6], получим формулу для искомой константы $\rho_{J \vartheta}$ в мезомолекуле $t t \mu$ для возбужденных состояний с $J=1$.

\section{3. АСИМПТОТИКА ВОЛНОВОЙ ФУНКЦИИ

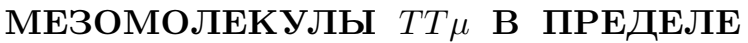 МАЛЫХ МЕЖЪЯДЕРНЫХ РАССТОЯНИЙ}

\section{В АДИАБАТИЧЕСКОМ ПРЕДСТАВЛЕНИИ ЗАДАЧИ ТРЕХ ТЕЛ}

В адиабатическом представлении задачи трех тел [7], [8] в пределе $R \rightarrow 0(r>R)$ объединенного атома волновая функция трех кулоновских частиц $t t \mu$ в возбужденном вращательном состоянии $J=1$ принимает вид [6]

$$
\Psi_{m J}^{J}(\mathbf{r}, \mathbf{R})=R^{-1} \sum_{N, l} \mathcal{R}_{N l}(r) \sum_{m=0}^{J} \Psi_{m m_{J}}^{l J}(\vartheta, \varphi, \theta, \phi) \chi_{N l m}^{J}(R) .
$$

Здесь $\mathcal{R}_{N l}(r)$ - радиальная кулоновская функция водородоподобного атома с зарядом ядра $z=z_{t}+z_{t}=+2 e$, функции $\chi_{N l m}^{J}(R)$ описывают относительное движение ядер трития в мезомолекуле $t t \mu$, причем в суммировании по квантовым числам $N, l, m$ присутствуют лишь четные $l$ начиная с $l=0$ вследствие симметрии волновой функции мезомолекулы, $l$ - квантовые числа оператора углового момента мюона, которые при фиксированном $J$ определяются правилом векторного сложения $\mathbf{J}=\mathbf{L}+\mathbf{l}$. Угловая часть $\Psi_{m m_{J}}^{l J}(\vartheta, \varphi, \theta, \phi)$ определена соотношением

$$
\begin{aligned}
& \left\langle\vartheta \varphi \theta \phi \mid l m J m_{J}\right\rangle \equiv \Psi_{m m_{1}}^{l J}(\vartheta, \varphi, \theta, \phi)= \\
& \quad=\frac{1}{\sqrt{2\left(1+\delta_{0 m}\right)}}\left\{Y_{l m}(\vartheta, \varphi) D_{m m_{J}}^{J}(\phi, \theta, 0)+Y_{l-m}(\vartheta, \varphi) D_{-m m_{J}}^{J}(\phi, \theta, 0)\right\},
\end{aligned}
$$

где $Y_{l m}(\vartheta, \varphi)$ - сферические гармоники, $D_{m m_{J}}^{J}(\phi, \theta, 0)$ - нормированные $D$-функции Вигнера, а $m_{J}$ - собственные значения оператора проекции $J_{Z}$ полного момента импульса мезомолекулы $\mathbf{J}$ на ось $O Z$ лабораторной системы координат, в которой направление вектора $\mathbf{R}$, соединяющего ядра трития, задается углами $\theta$ и $\phi$. Углы $\vartheta$ и $\varphi$ задают угловые координаты радиус-вектора мюона $\mathbf{r}$ во вращающейся системе координат, жестко связанной с межъядерной осью $R$, а квантовое число $m$ является собственным значением оператора проекции $l_{z}$ момента импульса мюона $\mathbf{l}$ на ось $O z$, совпадающую с межъядерной осью $R$ вращающейся системы координат.

Здесь следует отметить важное обстоятельство, которое состоит в том, что асимптотика (8) получена формальным предельным переходом $R \rightarrow 0(r>R)$ для волновой функции мезомолекулы $\Psi_{m_{1}}^{1}(\mathbf{r}, \mathbf{R})$, записанной в адиабатическом представлении задачи трех тел:

$$
\begin{gathered}
\Psi_{m_{1}}^{1}(\mathbf{r}, \mathbf{R})=\frac{1}{R} \sum_{j, m} \frac{1}{\sqrt{2\left(1+\delta_{0 m}\right)}}\left\{\psi_{j m}(\mathbf{r} ; R) D_{m m_{1}}^{1}(\phi, \theta, 0)+\right. \\
\left.+\psi_{j-m}(\mathbf{r} ; R) D_{-m m_{1}}^{1}(\phi, \theta, 0)\right\} \chi_{j m}^{1}(R),
\end{gathered}
$$


в котором базисные функции $\psi_{j m}(\mathbf{r} ; R)$ (решения квантово-механической задачи двух центров [25]) заданы во вращающейся системе координат, связанной с межъядерной осью $R$. Однако в пределе объединенного атома $R \rightarrow 0(r>R)$ межъядерная ось (ось $O z$ вращающейся системы координат) исчезает, и возникает вопрос согласования асимптотики (8) волновой функции задачи трех тел в адиабатическом представлении с физическими граничными условиями в этом пределе. Правильные физические условия в пределе объединенного атома можно задать в лабораторной системе координат, в которой угловые переменные радиус-вектора мюона $\mathbf{r}$ обозначим как $\widetilde{\vartheta}$ и $\widetilde{\varphi}$. Тогда угловая часть волновой функции мезомолекулы в лабораторной системе координат принимает вид

$$
\widetilde{\Psi}_{m_{1}}^{(l L) 1}(\widetilde{\vartheta}, \widetilde{\varphi}, \theta, \phi)=\sum_{m_{l}=-l}^{l} C_{l m_{l} L m_{L}}^{1 m_{1}} Y_{l m_{l}}(\widetilde{\vartheta}, \widetilde{\varphi}) Y_{L m_{L}}(\theta, \phi),
$$

где $C_{l m_{l} L m_{L}}^{1 m_{1}}$ - коэффициенты Клебша-Гордона. Функция $\widetilde{\Psi}_{m_{1}}^{(l L) 1}(\widetilde{\vartheta}, \widetilde{\varphi}, \theta, \phi)$ связана с функцией $\Psi_{m m_{1}}^{l 1}(\vartheta, \varphi, \theta, \phi)$, заданной во вращающейся системе координат, ортогональным преобразованием Чэнга-Фано [26]

$$
\Psi_{m m_{1}}^{l 1}(\vartheta, \varphi, \theta, \phi)=\sum_{L=|1-l|}^{1+l} \widetilde{\Psi}_{m_{1}}^{(l L) 1}(\widetilde{\vartheta}, \widetilde{\varphi}, \theta, \phi) G_{m L}^{l 1}
$$

где

$$
G_{m L}^{l 1}=(-1)^{l+m} \frac{1+(-1)^{l+L}}{\sqrt{2\left(1+\delta_{m 0}\right)}} C_{l m 1-m}^{L 0}
$$

Из соотношения (12) следует важное свойство преобразования $G_{m L}^{l 1}$ : оно диагонализует кориолисово взаимодействие

$$
\left\langle l m 1 m_{1}\left|(\mathbf{1}-\mathbf{l})^{2}\right| l m^{\prime} 1 m_{1}\right\rangle=\sum_{L=|1-L|}^{1+l} G_{m L}^{l 1} L(L+1) G_{m^{\prime} L}^{l 1},
$$

поскольку последнее возникает как следствие перехода из лабораторной системы координат во вращающуюся систему координат при описании системы трех тел.

Подставляя (12) в соотношение (8), находим асимптотику волновой функции мезомолекулы $t t \mu$ в пределе объединенного атома в лабораторной системе координат:

$$
\Psi_{m_{1}}^{1}(\mathbf{r}, \mathbf{R})=R^{-1} \sum_{N, l} \mathcal{R}_{N l}(r) \sum_{L=|1-l|}^{1+L} \widetilde{\Psi}_{m_{1}}^{(l L) 1}(\widetilde{\vartheta}, \widetilde{\varphi}, \theta, \phi) \sum_{m=0}^{1} G_{m L}^{l 1} \chi_{N l m}^{1}(R) .
$$

Далее задача сводится к нахождению асимптотики коэффициентов $\chi_{N l m}^{1}(R)$ в адиабатическом разложении (8) в пределе $R \rightarrow 0$. 
3.1. Асимптотика $\chi_{N l m}(R)$ при $R \rightarrow 0$. Коэффициенты $\chi_{N l m}^{1}(R)$ находятся при решении системы обыкновенных дифференциальных уравнений (в единицах $e=\hbar=m_{a}=1, m_{a}^{-1}=m_{\mu}^{-1}+M_{t}^{-1}, m_{\mu}$ - масса мюона)

$$
\begin{gathered}
\left\{\left(\frac{d^{2}}{d R^{2}}+2 M E_{J v}\right)-U_{i i}^{J}(R)\right\} \chi_{i}^{J}(R)=\sum_{j \neq i} U_{i j}(R) \chi_{j}^{J}(R), \\
U_{i i}^{J}(R)=\frac{J(J+1)-2 m^{2}}{R^{2}}+\frac{2 M}{R}+U_{i i}(R)
\end{gathered}
$$

с граничными условиями

$$
\chi_{i}(0)=\chi_{i}\left(R_{m} \rightarrow \infty\right)=0
$$

и условием нормировки

$$
\sum_{i} \int_{0}^{R_{m}} \chi_{i}^{2}(R) d R=1
$$

Здесь $M=M_{t} / 2$ - приведенная масса ядер трития, $U_{i j}(R)$ - эффективные потенциалы задачи трех тел (матричные элементы гамильтониана задачи трех тел), вычисленные в работах [27], [28] в адиабатическом представлении задачи трех тел, $\sum_{j \neq i}$ означает суммирование по дискретному $(\mathrm{Nlm})$ и непрерывному $(k l m)$ спектрам задачи двух кулоновских центров [25].

В работе [6] асимптотика решений $\chi_{i}(R)$ системы уравнений $(15)$ при $R \rightarrow 0$ была найдена с помощью преобразования Чэнга-Фано [26], которое связывает между собой угловые части волновой функции системы трех тел в лабораторной и вращающейся системах координат. Следуя идее этой работы, кратко изложим здесь методику нахождения главного члена асимптотики функций $\chi_{j}^{J}(R \rightarrow 0)$ для мезомолекулы $t t \mu$ с орбитальным моментом $J=1$.

В работе [6] было показано, что, сохраняя главный член асимптотики эффективных потенциалов [29], систему (15) при $R \rightarrow 0$ можно свести к виду

$$
\sum_{m^{\prime}}\left\{\delta_{m m^{\prime}} \frac{d^{2}}{d R^{2}}-\frac{1}{R^{2}}\left\langle i m J m_{J}\left|(\mathbf{J}-\mathbf{l})^{2}\right| i m^{\prime} J m_{J}\right\rangle\right\} \chi_{i m^{\prime}}^{J}(R)=0
$$

где $0 \leqslant\left(m, m^{\prime}\right) \leqslant \min (l, J)$, а матрица $R^{-2}\left\langle i m J m_{J}\left|(\mathbf{J}-\mathbf{l})^{2}\right| i m^{\prime} J m_{J}\right\rangle$ кориолисова взаимодействия диагонализуется с помощью ортогонального преобразования Чэнга-Фано:

$$
\left\langle i m J m_{J}\left|(\mathbf{J}-\mathbf{l})^{2}\right| i m^{\prime} J m_{J}\right\rangle=\sum_{L=|J-l|}^{J+l} G_{m L}^{l J} L(L+1) G_{m^{\prime} L}^{l J} .
$$

Учитывая это обстоятельство, ищем решение системы уравнений (16) в виде

$$
\chi_{i m}^{J}(R)=\sum_{\alpha} G_{m \alpha}^{l J} R^{\alpha+1} B_{N l}^{\alpha J}
$$


где $B_{N l}^{\alpha J}$ - искомые неопределенные коэффициенты. Подставив (18) в систему уравнений (16) и учитывая (17), а также свойство ортогональности преобразования $G_{m L}^{l J}$, получаем

$$
G_{m \alpha}^{l J}\{\alpha(\alpha+1)-L(L+1)\}=0
$$

откуда находим $\alpha=L$ для регулярного решения при $R \rightarrow 0$ и $\alpha=-(L+1)$ для нерегулярного решения. Поскольку нас интересует лишь регулярное при $R \rightarrow 0$ решение, асимптотику функций $\chi_{N l m}^{J}(R)$ представим в виде

$$
\chi_{N l m}^{J}=\sum_{L=|J-l|}^{J+l} G_{m L}^{l J} R^{L+1} B_{N l}^{L J} .
$$

Отсюда находим главный член асимптотики при $R \rightarrow 0$ для функций $\chi_{N l m}^{J}(R)$ :

$$
\chi_{N l m}^{J}(R)=G_{m|J-l|}^{l J} R^{|J-l|+1} B_{N l}^{|J-l| J}
$$

где константу $B_{N l}^{|J-l| J}$ можно определить по формуле

$$
B_{N l}^{|J-l| J}=\frac{1}{G_{m|J-l|}^{l J}} \lim _{R \rightarrow 0} \frac{\chi_{N l m}^{J}(R)}{R^{(|J-l|+1)}},
$$

используя численное решение системы дифференциальных уравнений (15). Здесь следует подчеркнуть то обстоятельство, что для получения правильной зависимости $\chi_{N l m}^{J}(R)$ от $R$ при $R \rightarrow 0$ необходимо удерживать в разложении (8) по адиабатическому базису все базисные функции с $m \leqslant \min (l, J)$.

3.2. Вычисление интеграла $\rho_{J \vartheta}$ в уравнении $(7)$ для мезомолекулы $t t \mu$. Подставив найденную асимптотику (20) для функции $\chi_{N l m}^{J}(R)$ в формулу $(14)$ и воспользовавшись свойством ортогональности преобразования $G_{m 0}^{l J}$, найдем асимптотику волновой функции мезомолекулы $t t \mu$ в состоянии $J=1$ при $R \rightarrow 0(r>R)$ :

$$
\Psi_{m_{1}}^{1}(\mathbf{r}, \mathbf{R})=\mathrm{R}_{10}(r) Y_{00}(\widetilde{\vartheta}, \widetilde{\phi}) Y_{1 m_{1}}(\theta, \phi) B_{10}^{11} R
$$

Используя полученное выражение, вычислим интеграл $\rho_{J=1, \vartheta}$, необходимый для нахождения скоростей ядерных реакций (7):

$$
\rho_{J \vartheta}=\lim _{R \rightarrow 0} \int \overline{\left|\frac{d}{d R} \Psi^{J \vartheta}(\mathbf{r}, \mathbf{R})\right|^{2}} d^{3} r=\frac{1}{4 \pi}\left[B_{10}^{11}\right]^{2},
$$

где $B_{10}^{11}=\lim _{R \rightarrow 0} \chi_{100}^{1 \vartheta}(R) / R^{2}$ (при получении $B_{10}^{11}$ было учтено, что в формуле $(21)$ $\left.G_{11}^{01}=1\right)$. Для мезомолекулы $t$ t $\mu$ имеем $\rho_{10}=5.23 \times 10^{-17} \mathrm{\phi м}^{-5}$ и $\rho_{11}=4.83 \times$ $10^{-17} \mathrm{\phi m}^{-5}$. 


\section{4. ЗАКЛЮЧЕНИЕ}

Найденная асимптотика (22) волновой функции трехтельной кулоновской системы $t t \mu$ была успешно использована для расчета скоростей ядерных реакций $\lambda_{f}^{J \vartheta}$ из возбужденных состояний этой мезомолекулы с орбитальным моментом $J=1$. Используя выражение (23) для интеграла $\rho_{J \vartheta}$ в формуле $(7)$, находим

$$
\lambda_{f}^{J \vartheta}=\frac{4}{3} K_{1} \rho_{J \vartheta}
$$

Здесь множитель 4/3 учитывает то обстоятельство, что в мезомолекуле ядерная реакция идет из определенного спинового состояния $I$ ядер трития $\left(I=S_{t}+S_{t}=1\right.$ для $J=1)$, а константа $K_{1}$ ядерной реакции в р-волне между ядрами трития "на лету" извлекалась из экспериментальных данных по сечениям $t t$-реакции, полученным с неполяризованными ядрами трития.

Формула (24) оказалась очень востребованной в связи с работами по мюонному катализу, где разработанная экспериментальная методика позволила измерить напрямую скорость ядерной реакции в мезомолекуле $t t \mu$ из возбужденных состояний с орбитальным моментом $J=1: \lambda_{f}^{\exp }=(15 \pm 2) \times 10^{6} \mathrm{c}^{-1}[13]$ и $\lambda_{f}^{\exp }=$ $(15.6 \pm 2.0) \times 10^{6} \mathrm{c}^{-1}[15],[16]$. Эти значения оказались очень близкими к нашим расчетам $\lambda_{f}^{\text {theor }}=13 \times 10^{6} \mathrm{c}^{-1}[20]-[22]$, выполненным в адиабатическом представлении задачи трех тел по изложенной выше схеме. Удивительно, но более поздние вариационные расчеты волновых функций $t t \mu$, выполненные группой Монхорста [18], и расчеты с использованием разложения по гауссовому базису, выполненные группой Камимуры [30], дают значения интегралов $\rho_{J=1, \vartheta}$, в несколько раз превышающие вычисленное нами значение [20], [22] и, как следствие, приводящие к существенному превышению рассчитанной скорости ядерной реакции $\lambda_{f}^{\text {theor }}$, экспериментального значения $\lambda_{f}^{\exp }$ и нашего расчета величины $\lambda_{f}^{\text {theor}}$, выполненного в адиабатическом представлении задачи трех тел.

Во всех этих расчетах использовалась одна и та же константа ядерной реакции (6), извлекаемая путем экстраполяции дифференциальных сечений $t t$-реакции "на лету" к нулевой энергии относительного движения ядер. Для этой реакции прямой расчет ядерной константы $K_{1}$, в отличие от малочастичной трехнуклонной системы $p d$ [2], в настоящее время невозможен, а надежная экспериментальная информация по угловому распределению продуктов реакции в низкоэнергетической области отсутствует. Действительно, константа $K_{1}=4.3 \times 10^{23}$ фм $^{5} \mathrm{c}^{-1}$ была вычислена на основе экспериментальных данных по дифференциальному сечению $t t$-реакции при $E=0.65 \mathrm{MэB,} \mathrm{приведенных} \mathrm{в} \mathrm{работе} \mathrm{[24].} \mathrm{Найденное} \mathrm{значение} \mathrm{согласуется}$ с оценкой, полученной в работе [25] для коэффициента анизотропии в $t t$-реакции при существенно более низкой энергии $(E=0.04 \mathrm{MэB})$, где, однако, экспериментальные ошибки составляют $\sim 50 \%$.

С момента выполнения работ [24], [25] ситуация по экспериментальному изучению $t t$-реакции "на лету" не улучшилась [31], однако достигнут существенный прогресс в измерении скоростей ядерных реакций в мезомолекулах $\lambda_{f}^{J \vartheta}$, в частности в мезомолекуле $t t \mu[16]$. Это позволяет рассчитывать константы ядерных реакций $K_{1}$ по формулам типа $(24),(23)$, используя измеренные значения $\lambda_{f}^{J \vartheta}$ и асимптотику 
волновых функций мезомолекул в пределе объединенного атома вместо малоэффективной при низких энергиях процедуры извлечения $K_{1}$ из экспериментальных данных по ядерным реакциям синтеза "на лету". В этой связи найденная в рамках адиабатического представления асимптотика волновых функций мезомолекул в пределе объединенного атома может быть использована в качестве "контрольной точки" при уточнении интегралов $\rho_{J \vartheta}$ в рамках альтернативных подходов, например вариационного [19] или адиабатического гиперсферического подходов [17]. Нахождение констант ядерных реакций синтеза является актуальной задачей для построения различных моделей в ядерной астрофизике [31].

\section{Список литературы}

[1] V. V. Gusev, I. V. Puzynin, V. V. Kostrykin, A. A. Kvitsinsky, S. P. Merkuriev, L. I. Ponomarev, Few-Body Systems, 9:2-3 (1990), 137-153.

[2] L. N. Bogdanova, Yu. A. Kuperin, A. A. Kvitsinsky, V.E. Markushin, S. P. Merkuriev, L. I. Ponomarev, Muon Catalyzed Fusion, 3 (1989), 377-387.

[3] Я. Б. Зельдович, Докл. АН СССР, 95 (1954), 493-495.

[4] Я. Б. Зельдович, С. С. Герштейн, УФН, 71:8 (1960), 581-630.

[5] J. D. Jackson, Phys. Rev., 106:2 (1957), 330-339.

[6] С. И. Виницкий, В. С. Мележик, Л. И. Пономарёв, ЯФ, 36:2(8) (1982), 465-473.

[7] С. И. Виницкий, Л. И. Пономарёв, ЯФ, 20:3 (1974), 576-588.

[8] С. И. Виницкий, Л. И. Пономарёв, ЭЧАЯ, 13:6 (1982), 1336-1418.

[9] Л.Н. Богданова, В.Е. Маркушин, В.С. Мележик, Л. И. Пономарёв, ЯФ, 34:6(11) (1981), 1191-1206.

[10] Л.Н.Богданова, В. Е. Маркушин, В. С. Мележик, ЖЭЭТ, 81:3 (1981), 829-841.

[11] L. N. Bogdanova, V.E. Markushin, V.S. Melezhik, L. I. Ponomarev, Phys. Lett. B, 115:3 (1982), 171-173; Phys. Lett. B, 167:4 (1986), 485-486.

[12] D. V. Balin, E. M. Maev, V. I. Medvedev, G. G. Semenchuk, Yu. V. Smirenin, A. A. Vorobyov, An. A. Vorobyov, Yu. K. Zalite, Phys. Lett. B, 141:3-4 (1984), 173-176.

[13] W. H. Breunlich, M. Cargnelli, P. Kammel et al., Muon Catalyzed Fusion, 1:1-3 (1987), $121-129$.

[14] T. Matsuzaki, K. Nagamine, K. Ishida et al., Hyperfine Interactions, 118:1-4 (1999), 229-234.

[15] L. N. Bogdanova, V.R. Bom, A. M. Demin et al., ЖЭТФ, 135:2 (2009), 242-252.

[16] Л.Н.Богданова, Д. Л. Дёмин, В. В. Фильченков, ЯФ, 78:1-2 (2015), 12-22.

[17] D. I. Abramov, L. N. Bogdanova, V. V. Gusev, L. I. Ponomarev, Hyperfine Interactions, 101:1 (1996), 301-306.

[18] S. A. Alexander, P. Froelich, H. J. Monkhorst, Phys. Rev. A, 41:5 (1990), 2854-2857; Erratum, 43:5 (1991), 2585.

[19] L. N. Bogdanova, V.I. Korobov, L. I. Ponomarev, Hyperfine Interactions, 118:1 (1999), $183-186$.

[20] В. С. Мележик, Автореферат диссертации на соискание ученой степени кандидата физ.-матем. наук, Препринт Р-81-463, ОИЯИ, Дубна, 1981.

[21] L. N. Bogdanova, V.E. Markushin, V.S. Melezhik, "Abstract", International Symposium on Muon Catalyzed Fusion ( $\mu$ CF-87), Nuclear Physics Institute, Leningrad, 1987, 147.

[22] L. N. Bogdanova, Muon Catalyzed Fusion, 3 (1988), 359-376.

[23] H. M. Agnew, W. T. Leland, H. V. Argo, R. W. Crews, A. H. Hemmendinger, W. E. Scott, R. F. Taschek, Phys. Rev., 84:4 (1951), 862-863.

[24] В. И. Серов, С. Н. Абрамович, Л. А. Моркин, Атомная энергия, 42 (1977), 59-61. 
[25] И. В. Комаров, Л. И. Пономарёв, С. Ю. Славянов, Сфероидальные и кулоновские сфероидальные функиии, Наука, М., 1976.

[26] E. S. Cheng, U. Fano, Phys. Rev. A, 6:1 (1972), 173-185.

[27] L. I. Ponomarev, T. P. Puzynina, N. F. Truskova, J. Phys. B, 11:22 (1978), 3861-3874.

[28] L. I. Ponomarev, T. P. Puzynina, L. N. Somov, J. Phys. B, 10:7 (1977), 1335-1343.

[29] M. P. Faifman, L. I. Ponomarev, S. I. Vinitsky, J. Phys. B, 9:13 (1976), 2255-2268.

[30] E. Hiyama, M. Kamimura, Y. Hamahata, Y. Kino, RIKEN Rev., 20 (1999), 34.

[31] С. Н. Абрамович, С. М. Таова, Изв. РАН. Сер. Физ., 76:4 (2012), 446-452. 